\title{
Efeito de um programa de exercício aquático na pressão arterial de idoso com hemiparesia
}

\author{
Alisson Padilha de Lima*, Fabrício Bruno Cardoso"*
}

\section{Resumo}

Este trabalho se propõe a avaliar o efeito de um programa de exercício aquático e seus benefícios sobre a pressão arterial de idoso com hemiparesia. O caso estudado foi de um idoso com idade de 60 anos que apresentava hemiparesia do lado esquerdo. Como estratégia metodológica de intervenção, utilizou-se de um programa de exercício aquático, e como estratégia de avaliação, foi aferida a pressão arterial, em conformidade com os procedimentos indicados pelo III Consenso Brasileiro de Hipertensão Arterial, ou seja, antes e após a prática de exercícios. Essa atividade ocorreu num período de cinco meses, com frequência de cinco vezes semanais, totalizando 90 sessões de exercícios, sendo que, cada sessão tinha duração de uma hora e ocorria de forma individualizada. Por meio dos dados obtidos, o estudo apresentou uma média de $150 \mathrm{mmHg}$ no primeiro mês, e, no último mês avaliado, um escore de $125 \mathrm{mmHg}$ na pressão sistólica, e na diastólica uma média de $95 \mathrm{mmHg}$ no primeiro mês. Além disso, um escore de 80 $\mathrm{mmHg}$ no último mês avaliado, mostrando a eficácia da intervenção proposta. Dessa forma, conclui-se que as intervenções executadas por intermédio do programa de exercício aquático foram benéficas sobre os níveis de pressão arterial sistólica e diastólica do idoso com hemiparesia, tendo importante papel na conscientização e na profilaxia da população que apresenta essa patologia, como um método eficaz aos fatores de risco a possíveis ataques sistêmicos no decorrer da vida.

Palavras-chave: Atividade física. Acidente vascular encefálico. Pressão arterial. Terceira idade.

\section{Introdução}

Alguns índices mundiais apontam a
elevação da pressão arterial (PA) e con-
sequentemente, a hipertensão, como um
dos maiores indicadores de mortalidade.
Dados apontam 7,1 milhões de mortes
anualmente, identificando cerca de $16 \%$
de doenças cardíacas isquêmicas, $21 \%$ de

* Educador Físico, Programa de Mestrado em Envelhecimento Humano - Universidade de Passo Fundo - UPF, bolsista Prosup/Capes, pesquisador do Laboratório de Temas Filosóficos em Conhecimento Aplicado (LABFILC) - Universidade Estadual do Rio de Janeiro (UERJ), Departamento de Ciências da Atividade Física. E-mail: professor.alissonpadilha@gmail.com

** Centro de Estudos em Neurociências e Educação (Neuroeduc/UFRJ), Programa de Doutorado em Neurobiologia do Instituto de Biofísica Carlos Chagas Filho (IBCCF/UFRJ), pesquisador do Laboratório de Temas Filosóficos em Conhecimento Aplicado (LABFILC) - Universidade Estadual do Rio de Janeiro (UERJ), Departamento de Ciências da Atividade Física. Email: fabriciobrunocardoso@gmail.com

$\rightarrow$ http://dx.doi.org/10.5335/rbceh.2012.3969

Recebido em: 14/05/2014. Aprovado em: 17/11/2014 
doenças vasculares periféricas e $24 \%$ de infarto agudo do miocárdio, casos esses atribuídos à hipertensão. Assim, devem ser mobilizados esforços para se prevenir tal ocorrência no processo de envelhecimento (SUBRAMANIAN et al., 2011).

O processo de envelhecimento humano tem sido caracterizado por diversas alterações biopsicossociais, ocasionando uma desestruturação orgânica na aptidão funcional e nas condições de saúde quando não adotado um estilo de vida saudável (LIMA; DELGADO, 2010). Já as alterações fisiológicas advindas da prática de exercício físico são responsáveis, no processo de senescência, pela melhora da qualidade de vida, da composição corporal, da aptidão funcional e das capacidades físicas inerentes para um envelhecimento ativo (LIMA et al., 2011). Além da melhora da capacidade funcional, um programa de exercício físico bem orientado oferece diversos benefícios no aumento das capacidades coordenativas e na saúde da população idosa (LIMA; CARDOSO, 2013).

Analisando os níveis de PA de um indivíduo idoso, têm se destacado, no cenário científico, as suas relações com doenças cardiovasculares. A PA tem fatores fisiológicos aos quais se deve atentar, como a variação em virtude da interação de fatores neuro-hormonais, comportamentais e ambientais, por exemplo (SBH, 2011). Portanto, a hipertensão pode desencadear patologias cardiovasculares como o acidente vascular encefálico (AVE), ocasionando sérias alterações na marcha, dentre elas, a hemiparesia, caracterizada pela perda de força muscular no domínio contrala- teral da lesão encefálica, resultando na limitação da realização de atividades da vida diária (AVD) e na diminuição da sua qualidade de vida (TYSON et al., 2006; CARR; SHEPHERD, 2008).

Dessa forma, existe uma variação contínua da PA, batimento a batimento, de acordo com as atividades de um indivíduo e, em hipertensos ou em pessoas acometidas com patologias como o AVE, essa variabilidade apresenta maior amplitude do que em normotensos. Ela está relacionada com um pior prognóstico, não se esquecendo de se atentar que durante o período de vigília esses valores são maiores do que os obtidos durante o sono, obtendo uma atenção maior para que se insiram intervenções profiláticas como a prática de exercício físico (GUPTA, 2004).

Nesses termos, o exercício aquático pode ser incluído como uma estratégia para contrabalançar o declínio físico e prevenir patologias como a hipertensão arterial (TOKMAKIDIS; SPASSIS; VOLAKLIS, 2008). Outros estudos têm comprovado a importância do exercício aquático na prevenção de diversas doenças, mas principalmente em condições clínicas específicas, como doenças arteriais coronarianas, fibromialgia e hipertensão arterial (MUNGUIA-IZQUIERDO; LEGAZ-ARRESE, 2008; LAURENT et al., 2009).

Estudos apontam que um indivíduo que adota um estilo de vida saudável, com a prática regular de exercício físico - especialmente em meio líquido, que tem por efeito benéfico seu ambiente como naturais controladores dos níveis pressóricos -, tem minimizado os riscos 
de patologias cardiovasculares como o $\mathrm{AVE}$, e tem maior controle dos níveis sistólicos e diastólicos da PA(SCHWARZ; HALLE, 2006; LIMA et al., 2010).

Portanto, por meio da hipótese elaborada, o presente estudo tem por objetivo avaliar o efeito de um programa de exercício aquático sobre a pressão arterial de um idoso com hemiparesia e seu efeito benéfico no controle dos níveis pressóricos.

\section{Material e métodos}

\section{Relato do caso}

Este estudo tem por característica um modelo de estudo de caso avaliativo, em que foram realizadas intervenções durante cinco meses sobre a evolução dos níveis pressóricos. O caso estudado foi de um paciente idoso, do sexo masculino, com idade de 60 anos, morador há 40 anos de uma cidade no interior do estado de Rondônia, que apresentava um quadro clínico de dois AVE, sérios déficits na marcha e hemiparesia do lado esquerdo, além de muita dificuldade na comunicação. O quadro clínico foi examinado por uma equipe multidisciplinar.

Conforme o histórico de saúde do paciente, retratado pelo seu médico, o tabagismo foi apontado pelas avaliações clínicas como um dos maiores causadores dessa patologia, juntamente com os hábitos alimentares inadequados, associados ao estilo de vida sedentário. $O$ paciente não praticava nenhum tipo de exercício físico regularmente, apresentando um grau de obesidade mórbida no decorrer das últimas décadas, o que afetou a sua qualidade de vida.
Antes do início das intervenções por parte do programa de exercícios aquáticos, o paciente realizou avaliações médicas e baterias de exames clínicos, como hemograma completo, lipidograma completo, urinálise e avaliações neurológicas que foram acompanhadas semanalmente, identificando o estado clínico do paciente e o modo como estava reagindo em meio às intervenções propostas. Após avaliado o seu estado de saúde e identificada sua normalidade nos exames clínicos, o paciente foi liberado para a prática do programa de exercícios aquáticos proposto neste estudo.

Além da supervisão do médico neurologista, o paciente teve acompanhamento de avaliações realizadas por uma equipe multidisciplinar composta por profissionais das áreas de Educação Física, Fisioterapia, Nutrição e Fonoaudiologia.

\section{Aspectos éticos}

A coleta dos dados se deu em conformidade com o disposto na resolução 196/96 do Conselho Nacional de Saúde. Inicialmente, foi solicitada autorização do participante para que o estudo fosse desenvolvido. Isso foi feito por meio de um Termo de Consentimento Livre e Esclarecido o TECLE, ao qual se anexou uma carta de esclarecimentos, explicando a natureza do estudo. $\mathrm{O}$ projeto desta pesquisa foi submetido ao Comitê de Ética em Pesquisa envolvendo Seres Humanos do Centro Universitário Luterano de Ji-Paraná CEULJI/ULBRA e aprovado com o protocolo $\mathrm{n}^{\mathrm{o}}$ 189/09. 


\section{Procedimentos de intervenção}

Como estratégia metodológica de intervenção, utilizou-se de um programa de exercícios aquáticos que foram desenvolvidos em uma piscina coberta $\mathrm{e}$ aquecida a uma temperatura que se considera adequada para esse tipo de trabalho, conforme citam Silva e Lima (2011), que é de $26^{\circ}$ a $30^{\circ}$ para que não haja desconfortos fisiológicos do indivíduo. As intervenções aconteceram num período de cinco meses, com frequência de cinco vezes semanais, totalizando noventa sessões de exercícios, sendo que cada sessão de exercício tinha duração de uma hora. As sessões foram compostas e subdivididas em aquecimento, parte principal com exercícios funcionais de predominância aeróbica, voltada especificamente para a melhora do condicionamento físico, com frequência de três vezes na semana e exercícios de fortalecimento muscular, duas vezes na semana, especificamente para a melhora da marcha do paciente.

Para a execução do programa de exercícios aquáticos, foram utilizados alguns equipamentos, tais como halteres e caneleiras próprias para exercício em meio líquido, flutuadores "espaguetes" como auxiliares de apoio ao paciente, conforme se utilizou o próprio peso corporal do aluno para exercícios funcionais e de fortalecimento.

O programa de exercícios foi proposto e dividido em sequência de execução:

1) Aquecimento: iniciou-se com caminhada para frente e para trás contra a resistência da água, deslocamento, utilizando a lateralidade; movimentos de rotação dos membros superiores; abdução e adução de membros superiores; prática de saltitar durante 2 minutos; movimentação de elevação de joelhos; e execução de movimentos de rotação dos pés. Esses exercícios foram executados como forma de preparação por meio do aumento da temperatura corporal para a execução de movimentos mais complexos;

2) Parte principal (exercícios de predominância aeróbica e exercícios de fortalecimento muscular): foram propostos exercícios funcionais específicos e adaptados de Lima et al. (2011), tais como transferência de peso em bipedestação, elevação de joelhos alternadamente, chutes para trás, chutes para frente, abdução e adução de membros inferiores, execução de movimento de chute com a perna reta. Como atividades aeróbicas, as práticas foram as de deambular mudando de direção e executando movimentos de flexão e extensão das pernas, saltitar, fazer movimentos cíclicos simulando o pedalar de uma bicicleta apoiado com flutuadores. Já para os exercícios de fortalecimento muscular, foram realizados movimentos similares aos utilizados por aparelhos específicos de academia, porém, usando o meio aquático. Assim, foram feitos movimentos de flexão e extensão de braços, abdução e adução de ombros, elevação frontal, elevação lateral, todos utilizando halteres. $\mathrm{E}$, para membros inferiores, foram 
feitos abdução e adução, flexão e extensão, movimentos rotatórios com os pés e elevação do peso corporal na ponta dos pés com caneleiras. Portanto, foi utilizada uma sequência de duas a três séries com oito a dez repetições na fase de adaptação e aumento progressivo de séries e repetições conforme a melhora do paciente, chegando ao final do programa com quatro séries de dez repetições. Foi monitorado o nível de intensidade por meio de comandos verbais, sempre em tom moderado para que não proporcionasse desconforto fisiológico ao paciente, como recomenda a American College of Sports Medicine (ACSM, 2011);

3) Volta calma ou relaxamento: foram executados alongamentos de membros superiores e membros inferiores seguidamente com relaxamento autógeno via respirações simultâneas, objetivando o controle interno do sistema orgânico (homeostase).

\section{Procedimentos de avaliação}

Como estratégia metodológica de avaliação deste estudo, utilizou-se a aferição da PA do indivíduo durante o período de intervenção desenvolvido, ou seja, durante a prática do referido programa de exercício aquático. A PA foi aferida antes de iniciar a prática, quando o aluno se posicionou sentado em um ambiente calmo e, após a realização dos exercícios, respeitando um intervalo necessário de cinco minutos para se constatar o efeito do exercício físico durante as noventa sessões, totalizando 180 aferições, por intermédio do aparelho esfigmomanômetro da marca (Welch allyn). Cabe ressaltar que se adotou todos os procedimentos indicados pelo III Consenso Brasileiro de Hipertensão Arterial (KOHLMANN JUNIOR et al., 1999).

\section{Análise estatística}

A análise dos dados deste estudo foi realizada com base na comparação de resultados estatísticos, utilizando-se o programa de estatística BIOESTAT 5.0. Os resultados obtidos pelas avaliações dos testes foram calculados por meio da estatística descritiva, a mediana e o teste para comparação Qui-quadrado.

\section{Resultados}

A Tabela 1 demonstra os níveis de variação da pressão arterial sistólica no primeiro mês, em que se obteve uma mediana de $150 \mathrm{mmHg}$, já no segundo e no terceiro mês, o sujeito apresentou uma mediana simultânea de $140 \mathrm{mmHg}$, tendo uma diminuição nos níveis pressóricos, o que ficou evidente no quarto mês, com um escore de $130 \mathrm{mmHg}$, e no quinto mês, com uma mediana de $125 \mathrm{mmHg}$. 
Tabela 1: Resumo descritivo dos níveis da pressão sistólica durante o período de intervenção

\begin{tabular}{|c|c|c|c|c|c|}
\hline Categorias & $1^{\circ}$ Mês & $2^{\circ}$ Mês & 3ํo Mês & $4^{\circ}$ Mês & 5ํMês \\
\hline № de Aferições & 36 & 36 & 36 & 36 & 36 \\
\hline Mínimo & 140.0 & 130.0 & 130.0 & 120.0 & 120.0 \\
\hline Máximo & 160.0 & 140.0 & 150.0 & 140.0 & 140.0 \\
\hline Mediana & 150.0 & 140.0 & 140.0 & 130.0 & 125.0 \\
\hline Coeficiente de variação & $3.6 \%$ & $1.7 \%$ & $4.2 \%$ & $5.2 \%$ & $4.9 \%$ \\
\hline
\end{tabular}

A partir do teste estatístico Qui-quadrado, percebe-se que a evolução comentada anteriormente não foi ao acaso e, sim, pelos efeitos positivos da prática constante do programa de exercício físico, pois, para um QUI= 119,82, obteve-se um $\mathrm{p}=0,01$.

Figura 1: Frequência absoluta dos níveis da pressão sistólica por mês

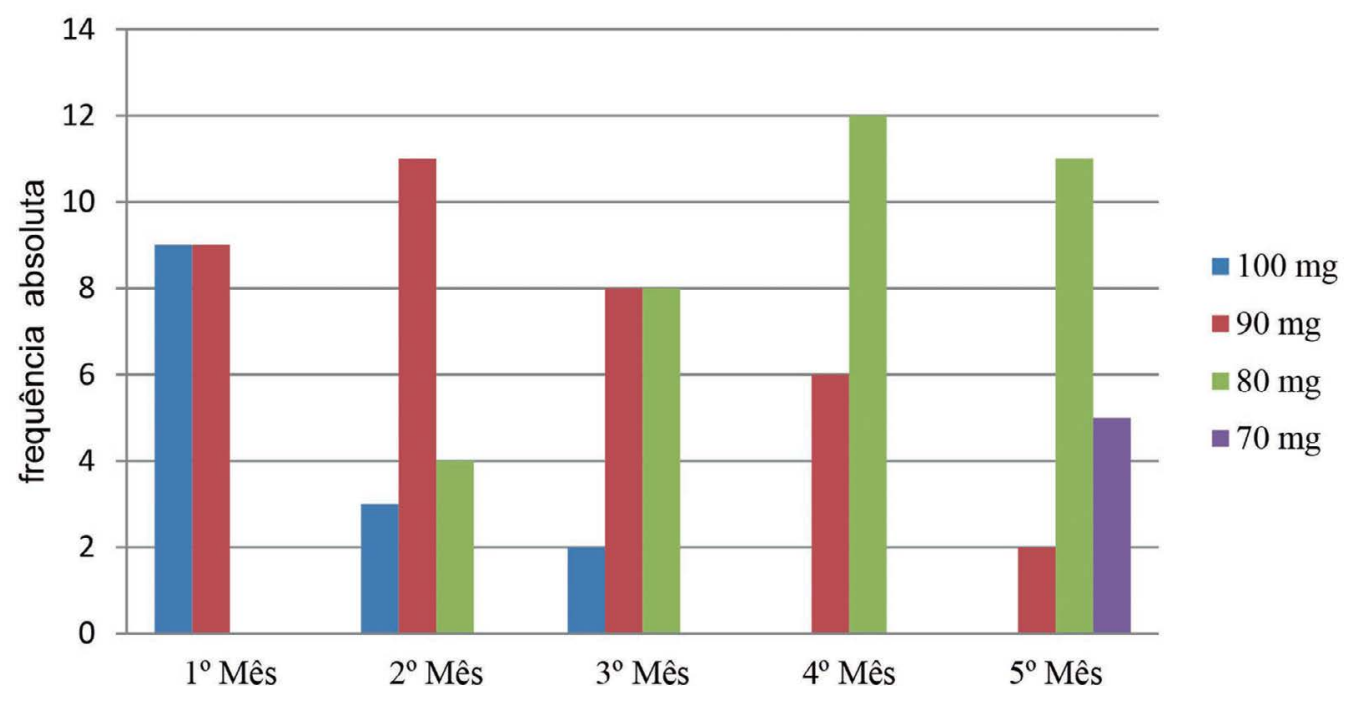

Com base nos dados da Tabela 2, de $90 \mathrm{mmHg}$, tendo um decréscimo nos percebe-se os níveis de variação da pres- níveis, o que vai ficando evidente no são arterial diastólica, que no primeiro quarto e no quinto mês, com um nível mês obteve uma mediana de $95 \mathrm{mmHg}$. de $80 \mathrm{mmHg}$, o que vem a comprovar a Já no segundo e no terceiro mês, o avaliaeficácia da intervenção proposta. do apresentou uma mediana simultânea 
Tabela 2: Resumo descritivo dos níveis da pressão diastólica durante o período de intervenção

\begin{tabular}{l|r|r|r|r|r}
\hline \multicolumn{1}{c|}{ Categorias } & \multicolumn{1}{c|}{ 10 Mês } & \multicolumn{1}{c|}{ 2 Mês } & \multicolumn{1}{c}{ 3o Mês } & \multicolumn{1}{c}{ 4 Mês } & \multicolumn{1}{c}{ 5o Mês } \\
\hline № de Aferições & 36 & 36 & 36 & 36 & 36 \\
Mínimo & 90.0 & 80.0 & 80.0 & 80.0 & 70.0 \\
Máximo & 100.0 & 100.0 & 100.0 & 90.0 & 90.0 \\
Mediana & 95.0 & 90.0 & 90.0 & 80.0 & 80.0 \\
Coeficiente de Variação & $5.4 \%$ & $7.1 \%$ & $7.9 \%$ & $5.8 \%$ & $7.9 \%$ \\
\hline
\end{tabular}

Ao estabelecer uma comparação a partir do teste estatístico Qui-quadrado, pode-se dizer que a intervenção desenvolvida neste estudo influenciou direta- mente na redução dos níveis da pressão diastólica do indivíduo participante nessa pesquisa, pois, para um QUI=60,35, obteve-se um $\mathrm{p}=0,03$.

Figura 2: Frequência absoluta dos níveis da pressão arterial diastólica por mês

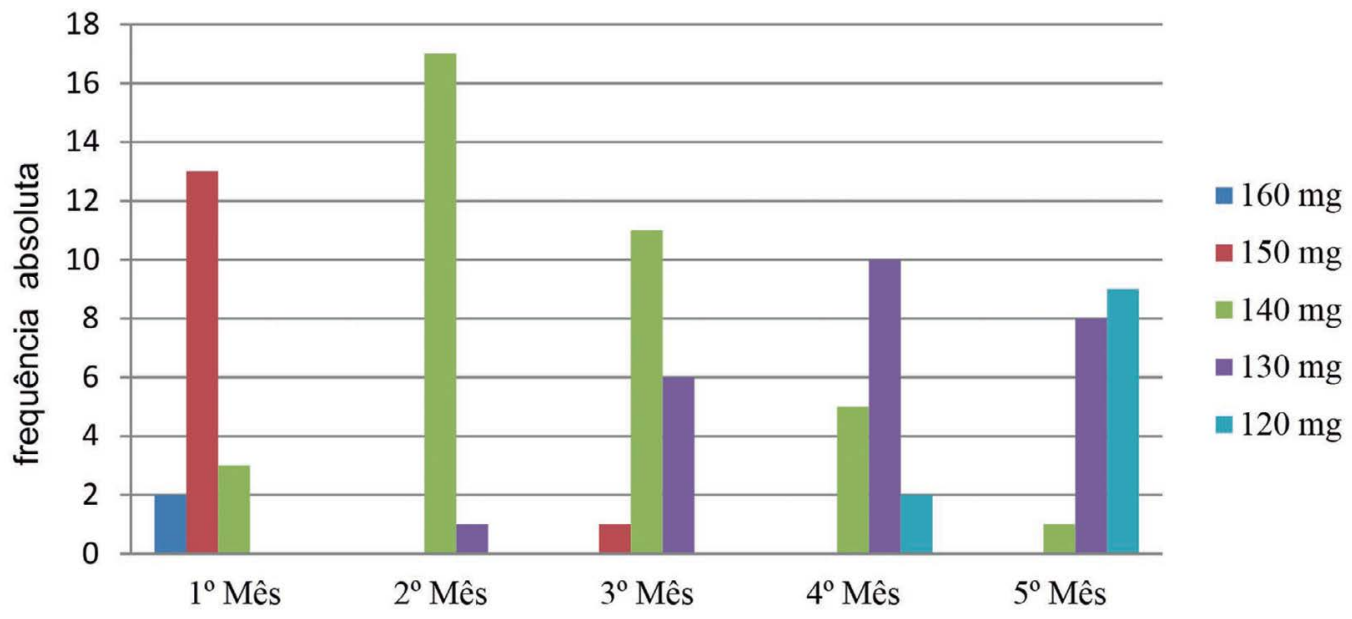

\section{Discussão}

Com os dados obtidos no presente estudo, pode-se constatar a eficácia da intervenção proposta, obtendo-se um bom escore dos níveis pressóricos sistólicos e diastólicos do caso avaliado, o que nos leva a analisar a eficácia que um programa de exercício aquático tem na profilaxia ou no tratamento não farmacológico da hipertensão arterial.

Tais informações se comprovam quando os números comparados ao estudo realizado por Mota et al. (2010) 
em indivíduos com marcha hemiparética após AVE, de forma que se constata que o exercício físico diminuiu a predisposição de ter um novo surto da doença, além de diminuir os níveis pressóricos e melhorar significativamente a sua capacidade aeróbica, efetivando, consequentemente, uma melhora na capacidade funcional da marcha.

Davis et al. (2013) afirmam em seu estudo que intervenções buscando a melhora das condições de saúde do idoso, como o treinamento aeróbico em meio líquido, podem proporcionar inúmeros benefícios no controle e na profilaxia de patologias como a hipertensão arterial. Esse, então, é considerado como um dos melhores tratamentos não farmacológicos para o controle dos níveis pressóricos e na minimização de possíveis fatores de risco cardiovasculares que podem levar ao AVE (SUBRAMANIAN et al., 2011).

Além de favorecer o controle dos níveis pressóricos, o exercício aquático pode propiciar melhoras na aptidão física, composição corporal e na capacidade cardiorrespiratória da população idosa, minimizando os efeitos deletérios do envelhecimento por meio dos benefícios proporcionados pela sua prática, em ambiente favorável e prazeroso de ser executado (BERGAMIN et al., 2013).

Conforme Hale, Waters e Herbison (2012), o exercício aquático é identificado em seu estudo como um dos métodos mais eficazes no trabalho de reabilitação de diversas patologias e na melhora da condição física da população idosa, como na diminuição de fatores de riscos agravantes para os risco à saúde, como hipertensão arterial e doenças osteoarticulares que incapacitam o indivíduo com sérios déficits da sua marcha, conforme o caso do presente estudo.

Como demonstrado no presente estudo, um indivíduo com níveis de PA elevados pode beneficiar-se consideravelmente com práticas regulares de um programa de exercícios aquáticos para atingir e manter uma redução dos níveis pressóricos (ACSM, 2011). Assim, o incentivo à constante prática de exercícios físicos, em diversos ambientes, em grupo isolado ou individualmente, sendo hipertenso ou não, proporciona um alto índice de profilaxia no tratamento não medicamentoso dessa patologia, e na melhora dos fatores de riscos à saúde, isso é, PA sistólica e diastólica, índice de massa corporal, frequência cardíaca, circunferência total da cintura, consumo máximo de oxigênio, melhora no nível do colesterol (SJOLING et al., 2011).

Torna-se evidente, assim, a eficácia da intervenção proposta no controle dos níveis pressóricos do indivíduo em estudo, evidenciando um cenário totalmente propício para o trabalho de reabilitação e melhora da mobilidade.

\section{Conclusão}

As intervenções executadas por intermédio do programa de exercício aquático foram eficazes sobre os níveis de pressão arterial sistólica e diastólica do idoso com hemiparesia, e podem ser consideradas importantes na conscientização e na profilaxia da população de risco que poderá apresentar essa patologia, como um método eficaz contra os fatores de risco a possíveis ataques sistêmicos no decorrer da vida. 
Devido à limitação de referências encontradas sobre a temática do estudo, recomenda-se que outros estudos sejam feitos, comparando o efeito do exercício aquático em idosos com hemiparesia com um número maior de participantes e de ambos os gêneros.

\section{Effect of a program of aquatic exercise on blood pressure in elderly with hemiparesis}

\section{Abstract}

Evaluate the effect of a program of aquatic exercise and its benefits on blood pressure in elderly patients with hemiparesis. The case studied was an elderly witch aged 60 years who had hemiparesis on the left side, as a methodological intervention strategy was used in a program of aquatic exercise and as an evaluation strategy to blood pressure was measured according to the procedures indicated by III Consensus Brazilian Hypertension before and after the practical exercises that took place over a period of five months to five times weekly, totaling 90 sessions of exercises, with each exercise session lasted for an hour individually. Through the study of the data shows an average of $150 \mathrm{mmHg}$ in the first month and last month reported a score of $125 \mathrm{mmHg}$ systolic and diastolic an average of $95 \mathrm{mmHg}$ in the first month and a score of $80 \mathrm{mmHg}$ in the last month reported showing the effectiveness of proposed intervention. Thus it is concluded that interventions implemented through the aquatic exercise program were beneficial on levels of systolic and diastolic blood pressure of elderly with hemiparesis, having important role in raising awareness and prophylaxis of the population has this disease, as an effective method the risk factors to potential systemic attacks throughout life.

Keywords: Physical activity. Stroke. Blood pressure. Seniors.

\section{Referências}

AMERICAN COLLEGE OF SPORTS MEDICINE- ACSM. Manual do ACSM para Avaliação da Aptidão Física Relacionada à saúde. 3. ed. Rio de Janeiro: Guanabara Koogan, 2011.

BERGAMIN, M. et al. Water-versus landbased exercise in elderly subjects: effects on physical performance and body composition. Clinical Interventions in Aging, Auckland, v. 8, p. 1109-1117, Aug. 2013.

CARR, J. H., SHEPHERD, R. B. Reabilitação neurológica: otimizando o desenvolvimento motor. São Paulo: Manole; 2008.

DAVIS, J. C. et al. Challenges with costutility analyses of behavioral interventions among older adults at risk for dementia. British Journal Sports Medicine, London, v. 9, p. 1-6, Nov. 2013.

HALE, L. A.; WATERS, D.; HERBISON, P. A randomized controlled trial to investigate the effects of water-based exercise to improve falls risk and physical function in older adults with lower-extremity osteoarthritis. Archives of Physical and Medicine Rehabilitation, Chicago, v. 93, n. 1, p. 27-34, Jan. 2012.

GUPTA, R. Trends in hypertension epidemiology in India. Journal of Human Hypertension, London, v. 18, n. 2, p. 73-78, July. 2004.

KOHLMANN JUNIOR, O. et al. III Consenso Brasileiro de Hipertensão Arterial. Arquivos Brasileiros de endocrinologia e metabologia, São Paulo, v. 43, n. 4, p. 257-286, Aug. 1999.

LAURENT, M. et al. Training--induced increase in nitric oxide metabolites in chronic heart failure and coronary artery disease: an extra benefit of water-based exer-cises? European Journal of Cardiovascular Prevention \& Rehabilitation, Oxford, v. 16, n. 2, p. 215-221, Apr. 2009. 
LIMA, A. P. et al. A importância do exercício físico para mulheres hipertensas. Efdeportes. com, (revista digital), Buenos Aires, v. 15, n. 147, p. 1-1, ago. 2010. Disponível em: <http:www. efdeportes.com>. Acesso em: 20 maio 2014.

LIMA, A. P.; CARDOSO, F. B. Avaliação da eficácia de um programa ludomotor de exercícios físicos na melhora da capacidade funcional de idosos. Estudos Interdisplinares sobre o envelhecimento, Porto Alegre, v. 18, n. 2, p. 429-440, dez. 2013.

LIMA, A. P.; DELGADO, E. I. A melhor idade do Brasil: aspectos biopsicossociais decorrentes do processo de envelhecimento. Ulbra e Movimento (REFUM), Ji-Paraná/RO, v. 1, n. 2, p. 76-91, set./dez. 2010.

LIMA, A. P. et al. Uma avaliação da eficácia de um programa neuromotor de exercícios físicos para idosos. Brazilian Journal of Biomotricity, Rio de Janeiro, v. 5, n. 1, p. 26-33, jan. 2011.

MOTA, R. S. et al. Avaliação da eficácia do exercício aeróbico na reabilitação de pacientes com marcha hemiparética. Fisioterapia Brasil, Rio de Janeiro, v. 11, n. 4, p. 259-264, ago. 2010.

MUNGUIA-IZQUIERDO, D.; LEGAZ-ARRESE, A. Assessment of the effects of aquatic therapy on global symptomatology in patients with fibromyalgia syndrome: a randomized controlled trial. Archives of Physical Medicine Rehabilitation, Chicago, v. 89, n. 12 , p. 2250-2257, Dec. 2008.

SBH, Sociedade Brasileira de Hipertensão. Diretrizes Brasileiras de monitorização ambulatorial (MAPA) e residencial (MRPA) da pressão arterial. Revista hipertensão, São Paulo, v. 14, n. 1, p. 6-11, jan. 2011.

SCHWARZ S, HALLE M. Blood pressure lowering through physical training-what can be achieved?. MMW Fortschritte der Medizin, München, v. 148, n. 47, p. 29-32, Nov. 2006.
SJOLING, M. et al. Effectiveness of motivational interviewing and physical activity on prescription on leisure exercise time in subjects suffering from mild to moderate hypertension. BMC Research Notes, London, v. 12 , p. 344-352, Sept. 2011.

SILVA, A. S. D.; LIMA, A. P. Os benefícios da reabilitação aquática para grupos especiais. EFDeports.com (revista digital), Buenos Aires, v. 16 , n. 160 , p. 1-1, Sept. 2011. Disponível em: <http:www.efdeportes.com>. Acesso em: 20 maio 2014.

SUBRAMANIAN, H. et al. Non-pharmacological Interventions in Hypertension: A Community-based Cross-over Randomized Controlled Trial. Indian Journal of Community Medicine, Mumbai, v. 36, n. 3, p. 191-196, July. 2011.

TYSON, S. F. et al. Balance disability after stroke. Physical Therapy, Alexandria, v. 86, n. 3, p. 30-8, July 2006.

TOKMAKIDIS, S. P.; SPASSIS, A. T.; VOLAKLIS, K. A. Training, detraining and retraining effects after a water-based exercise program in patients with coronary artery disease. Cardiology, New York, v. 111, n. 4, p. 257-264, Apr. 2008. 